ISSN 1996-1073

www.mdpi.com/journal/energies

Review

\title{
A Generic Framework for the Evaluation of the Benefits Expected from the Smart Grid
}

\author{
Spiros Livieratos ${ }^{1, *}$, Vasiliki-Emmanouela Vogiatzaki ${ }^{1}$ and Panayotis G. Cottis ${ }^{2}$ \\ 1 School of Pedagogical and Technological Education, Department of Electronic Engineering \\ Educators, Heraklion, Athens 14121, Greece; E-Mail: slvogiatzaki@hotmail.com \\ 2 School of Electrical and Computer Engineering, National Technical University of Athens, Iroon \\ Polytechniou 9, Athens 15780, Greece; E-Mail: pcottis@central.ntua.gr \\ * Author to whom correspondence should be addressed; E-Mail: slivieratos@aspete.gr; \\ Tel.: +30210-2896953; Fax: +30210-2896773.
}

Received: 23 November 2012; in revised form: 5 January 2013 / Accepted: 25 January 2013 / Published: 13 February 2013

\begin{abstract}
The Smart Grid has the potential to bring significant value to the various stakeholders of the electricity market. A methodology for the evaluation of the smart grid benefits is required to facilitate the decision making by quantifying the benefits expected from a smart grid project. The present paper proposes a generic framework to assess these expected benefits taking into account the regulatory, business and technical challenges focusing particularly on Distributed Systems Operators (DSOs) and end users. An indicative study case is presented where the proposed cost-benefit approach assesses the expected value of DSOs from the Smart Grid and determines whether and under what conditions such an investment should be initiated.
\end{abstract}

Keywords: smart grid; smart grid communications; grid reliability; benefits of smart grid; ICT technologies for smart grid

\section{List of Abbreviations:}

BN Business Need

CAIDI Customer Average Interruption Duration Index

CAPEX Capital Expenditure

DA Distribution and Automation

DG Distributed Generation

DGO Distributed Generation Operators 


$\begin{array}{ll}\text { DR } & \text { Demand Response } \\ \text { DSO } & \text { Distribution System Operators } \\ \text { EBIT } & \text { Earnings before Interests and Taxes } \\ \text { ETSP } & \text { Energy Transport service Providers } \\ \text { EV } & \text { Electric Vehicle } \\ \text { ICT } & \text { Information and Communication Technologies } \\ \text { IPP } & \text { Independent Power Producer } \\ \text { LV } & \text { Low Voltage } \\ \text { MV } & \text { Medium Voltage } \\ \text { NPV } & \text { Net Present Value } \\ \text { O\&M } & \text { Operation and Maintenance } \\ \text { OCF } & \text { Operating Cash Flows } \\ \text { OPEX } & \text { Operational Expenditure } \\ \text { PLC } & \text { Power Line Communications } \\ \text { QoS } & \text { Quality of Service } \\ \text { SAIDI } & \text { System Average Interruption Duration Index } \\ \text { SAIFI } & \text { System Average Interruption Frequency Index } \\ \text { SME } & \text { Small and Medium Enterprise } \\ \text { SOA } & \text { Service Oriented Architecture } \\ \text { T\&D } & \text { Transmission and Distribution } \\ \text { TSO } & \text { Transmission System Operators } \\ \text { WACC } & \text { Weighted Average Cost of Capital }\end{array}$

\section{Introduction}

The electric power industry is in the early stages of a complete transformation. A significant part of the existing electrical power infrastructure is worn out and must be replaced. Furthermore, the electric power industry must satisfy the increasing demand for new services and applications. As the electricity infrastructure must be upgraded and expanded, it will need enhanced monitoring, control and communication capabilities [1]. To incorporate new functions and services that range from the incorporation of intermittent, often distributed, renewable energy sources to the new and efficient ways that residential, commercial and industrial users consume electricity, the underlying Medium Voltage (MV) and Low Voltage (LV) power grid must be put through decisive changes. Though, traditionally, most parts of the electricity supply chain have been a state property, in the last 30 years governments started privatizing certain parts of the utilities. As a consequence of the withdrawal of natural monopolies from generation, electricity has become a commodity [2]. The functional separation of the vertically integrated electric utilities has also led to the development of new business entities and stakeholders, whereas [3] "Most of the problems that have arisen in electricity, such as those related to transmission congestion and peaking capacity, are attributable to specific flaws in the integrated spot market/dispatch process or to failures in taking full advantage of the spot prices arising from this 
process." This up to now slowly varying electric power transmission and distribution market is finding itself at the convergence of energy, information and communications technologies (ICT), necessitating drastic change and innovation to support the intelligent power grid of the 21 st century, namely the Smart Grid. The Smart Grid can be regarded as an ICT-enabled electric power network which can intelligently integrate/incorporate the actions of all the users connected to it, i.e., generators, distributors and consumers of energy, aiming at delivering electrical power in a sustainable, economic and secure way [4]. In addition to enhancing efficiency, reliability and safety, the Smart Grid will also enable: (i) the smooth integration of distributed generation (DG) of electric power including renewable sources, (ii) the practically real-time participation of energy producers and consumers into the energy market and (iii) the introduction of electrical vehicles (EV). All the above new services and/or enhanced applications necessitate ICT solutions intended specifically for the transmission and the distribution networks [5]. By properly exploiting its embedded ICT capabilities, the Smart Grid will enhance the Quality of Service (QoS) and optimize the network assets utilization, handle current and future demand, reduce costs for providers and consumers, implement disaster recovery plans and offer operational resilience and efficiency, enable almost real-time participation of consumers in the electricity market, incorporate all options for energy production and storage, encourage and facilitate the development of new products and services [6].

The present work deals with the key issues concerning the benefits expected from the Smart Grid and proposes a framework to assess these benefits from the perspective of the various entities involved. The first step of the proposed framework is to identify the key technical needs and business objectives that the electric power industry must address by employing the Smart Grid. Subsequently, these objectives are appropriately formulated, not necessarily in technical terms only, but also in non-technical terms expressing what should be accomplished. Next, a set of functions are proposed to describe the relation of the benefits to objectives taking into account that, though a smart grid functionality may originally be intended to offer a specific benefit, it may also offer additional benefits of various types.

The rest of the paper is structured as follows: in Section 2 the main objectives of the Smart Grid and the relevant benefits and benefited sectors involved are classified as well as a relevant literature overview is presented. In Section 3, regulatory, technical and business considerations related to the Smart Grid are discussed. In Section 4, a methodological framework is proposed for the assessment of the smart grid benefits whereas, in Section 5, a realistic study case is presented dealing with the evaluation of several primary reliability parameters and their economic impact to demonstrate how the proposed framework can be used.

\section{Objectives and Benefits of the Smart Grid}

The deployment of the Smart Grid must take into account numerous technical and commercial/business considerations as well as critical regulatory and environmental issues to conform to the international technical standards and regulations and satisfy particular social needs and governmental edicts. The above factors along with the evolution of ICT standards, services and products will determine the migration strategy from the conventional power grid to the Smart Grid as well as the relevant expected benefits. 
The Smart Grid will act as an intelligent agent creating economic value in the electricity market. The latter is divided into: (i) the wholesale electricity market, which will support the necessary changes in energy flows, introduce new areas for competition and revolutionize the way electricity is generated, transported and traded as Small and Medium Enterprises (SMEs) and households will become able to act as Independent Power Producers (IPPs) and (ii) the retail electricity market that reflects the final level of the electricity supply chain focusing on the interaction between suppliers and consumers of electricity, though certain aspects related to electricity supply such as metering are usually undertaken by DSOs [1].

\subsection{Targets of the Smart Grid}

The Smart Grid aims at:

(i) solving problems related to numerous grid operations such as demand/response (DR), automated measurement and control, grid monitoring and physical surveillance, real-time load management, power theft identification, etc.

(ii) ensuring interoperability and security of power supply;

(iii) integrating and managing and all kinds of distributed power generation including renewable energy sources;

(iv) leveraging the electricity market by allowing demand side participation, providing new tariff schemes and facilitating the consumers participation in the free energy market [7].

The benefits expected from the Smart Grid can be grouped into four basic categories: operational, economic, environmental/social and security-specific [8]. The specific benefits offered to DSOs and the end users are discussed below. The two lists are not exclusive noting that some benefits can be assigned to both [9].

\subsection{Benefits Offered to DSOs}

(i) Operational: The enhanced observability and manageability of the power system offered by the Smart Grid will allow DSOs to reduce the frequency and duration of power interruptions and outages, thus enhancing system reliability. Also, the preventive grid maintenance facilitated by the Smart Grid is expected to result in fewer component failures [9];

(ii) Economic: DSOs will benefit from the significant reduction offered by the Smart Grid both with regard to the technical losses on the distribution grid and to the peak load. Significant economic benefits will also be offered from leveraging the business opportunities brought in by the Smart Grid due to DR, microgrid operation, etc. In addition to the improved smart metering accuracy and the significant reduction of failures and outages, electricity theft will be drastically reduced and cash flows will improve as a result of timely billing and revenue collection;

(iii) Environmental/social: Lower losses on the transmission and distribution grid will come up due to network optimization. Also, the Smart Grid operation will enhance the seamless incorporation of closer-to-the-load power production from distributed renewable energy generation, thus reducing the proportion of fossil fuel based generation in the overall generation mix. This will result in fewer emissions due to the expected reduction in the use of 
fossil fuels. Moreover, the integration of intermittent renewable energy generators into the conventional power grid will also help the DSOs to improve their environmental profile by directing power generation towards environmentally friendly methods [10]. Also, the adaptation of power consumption to generation will be beneficial to DSOs since it is important to avoid reinforcement for the integration of photovoltaic and wind generators by reducing the feed-in peak. Through an intelligent and more efficient control of distributed energy resources, backup reserves and other ancillary services, the Smart Grid will maximize the power system capability to manage intermittent power generation [11,12];

(iv) Security-specific: The enhanced monitoring and physical surveillance of the electricity network will increase its robustness and resilience both from a physical and a cyber point of view.

\subsection{Benefits Offered to End Users}

(i) Operational: By enhancing the power system reliability, the Smart Grid will offer better QoS to the consumers. The consequent reduction in the frequency and duration of power interruptions and outages will be translated in less productivity and business losses for the consumers. Moreover, the ability to collect real-time information will give consumers the opportunity to control their consumption in practically real-time and engage themselves actively in the electricity market [8]. The significant peak load reduction expected from smart grid operation will allow the DSOs to reduce their costs and eventually the prices enjoyed by the consumers;

(ii) Economic: In addition to potential bill savings for all consumers, corporate users may enjoy significant indirect economic benefits as havoc situations that lead to severe losses of productivity will be prevented;

(iii) Environmental/social: Going green will be facilitated as the Smart Grid will enable the consumers to migrate to a more dynamic consumption pattern, thus indirectly leading to reduced energy consumption coming from fossil fuels. Being an essential component of the Smart Grid, smart metering will give the end-users the opportunity to control-practically in real time - the amount of energy they consume and how much they spend every month on their energy bills $[13,14]$. Demand-side management will enable consumers to adapt their energy consumption and, consequently, the level of the generated power. Moreover, shifting demand away from the peak will lower the peak prices $[11,12]$;

(iv) Security-specific: The Smart Grid will offer the end users enhanced protection against manmade attacks and natural disasters [11,12].

\section{Regulatory, Technical and Business Considerations Related to the Smart Grid}

\subsection{The General Regulatory Framework for DSOs}

DSOs usually operate in regulated markets $[15,16]$. Most regulators consider DSOs as natural monopolies that: (i) own and manage the MV and LV power grid and the related assets (e.g. power lines, transformers, switchgear, circuit breakers, sub-stations, meters, etc.) and (ii) guarantee the delivery of electric power to various kinds of end users. DSOs profit from charging system users (distributed generators - operators/retailers/consumers) for the transport of electric power from/to their 
premises. Also, the distributed energy generated is directly fed into the distribution network based on a (regulated) agreement between DSOs and the distributed generation operators (DGOs). The latter pay a connection charge and, sometimes, an additional use-of-system charge to the DSOs for the transport of electricity and other system services [17]. Tariffs for power distribution are regulated by the national regulatory authorities that define or approve the prices set by the DSOs. In general, DSOs are not permitted neither to own nor operate generation assets connected to their networks nor to engage in the retail market. As such, their function is quite different from that of other market stakeholders, namely generators, retailers/suppliers, energy service companies, which participate in free competitive markets.

Some regulatory frameworks do not allow DSOs to control load shifting/shedding, although it is of great interest to them [18]. Several activities - the so called ancillary services that, inter-alia, handle locally the real and reactive power-could be directly procured by DSOs but would require regulatory approval. In general, the development of the Smart Grid will require enhanced investment and innovation in the distribution grid, which will normally take place under regulated conditions. Thus, the regulatory framework must be reformulated to adapt to both new and reconfigured incentives taking into account the primary policy objectives related to electric power. In this course, regulation should incentivize innovations of grid operators when they implement assets related to the Smart Grid [19,20].

\subsection{Technical Considerations Related to the Smart Grid}

DSOs all over the world have acknowledged that the current ICT infrastructure of most distribution networks is - for the time being - insufficient for the deployment of the Smart Grid. The energy flow can not be fully monitored nor controlled since real-time communication either with distributed generators or with the end users is not possible [21].

\subsubsection{Smart Grid Communications}

Many DSOs have already deployed backbone fibre communication networks to monitor their crucial network operations, especially in the High Voltage networks [22]. On the other hand, DSOs have not yet deployed last mile communication networks to exchange information with the various nodes of the transmission and distribution grid. In addition to fibre communications, DSOs have other options to transfer data through the power network. Specifically, DSOs may use:

(i) Power Line Communications (PLC), a technology that moves digital information through the grid via the very cables used for the transmission and distribution of the electric power [23,24];

(ii) Wireless communication technologies, mainly for the last mile, to connect substations and provide services related to remote metering.

Reliable backbone communications infrastructure and last mile connectivity are of great interest to both DSOs and Telecom Companies (Telcos) since significant cost and investment savings are leveraged due to the drastic acceleration of the deployment of the Smart Grid.

The smart grid communications architecture involves two layers:

- $\quad$ The power system layer, responsible for the safety and reliability of the distribution network. 
- $\quad$ The communications layer intended for the exchange of information between any nodes of the power grid.

As reliability and resilience of the smart grid communications network constitute a major concern, especially to handle power supply interruptions and emergency situations or when alternative routing/transmission of critical data is not available/guaranteed, DSOs are extremely cautious about engaging third parties to provide, operate and/or own any part of the smart grid communications subsystems.

DSOs claim that they must own or, at least, fully control the smart grid communications infrastructure, which they should deploy and operate with strict resilience specifications, though, normally, the communications traffic moving through the network is relatively small $[4,25,26]$. Relying on external, non-dedicated communications infrastructure that might be offered by Telcos, is considered susceptible to severe risks.

\subsubsection{Smart Grid ICT Services}

The key functional requirements of the Smart Grid are related to reliability, security, interoperability, and operational and capital expenditures. These requirements will determine the ICT technology appropriate by case. Since almost all technologies and systems related to the Smart Grid already exist [21,25] the roll-out of smart grid projects does not require the development of new technologies but, instead, the interoperable incorporation of appropriate ICT solutions into the power system. However, prior to roll-out, important issues must be considered, particularly those related to capital investments and to the support, maintenance and lifetime of the power system.

Apart from the technical constraints related to the power grid infrastructure and topology, DSOs are also constrained by the international and national regulatory frameworks as well as by the specific business culture and modus operandi. On the other hand, the DSOs operation is influenced by the various stakeholders participating in the electric power market. These constraints/influences critically affect the selection of ICT solutions and the implementation of smart systems. It is unlikely that a single ICT solution will be applicable to or suitable for the entire power grid. This necessitates the standardization and interoperability of all types of information systems and communication platforms involved.

In addition to the appropriate communications infrastructure (wireless or wired), the deployment of the Smart Grid requires the definition of specific ICT services which may be classified into the following two major categories.

\subsubsection{Critical Services}

These are ICT services necessary for the operation of the electric power network in critical/emergency situations, for example to activate circuit breakers or to connect a back up power supply unit when necessary. For critical services requiring response times of the order of milliseconds, both DSOs and Telcos estimate that the existing ICT infrastructure is neither ubiquitous nor sufficiently robust/reliable [4,26]. Also, many current IP-based networking technologies are not suitable for smart grid critical services. Therefore, significant investment in advanced ICT 
infrastructure intended for the power grid is required. Taking into consideration that, on the one hand, it is mainly the regulated DSOs that will invest in the Smart Grid but, on the other hand, all the other stakeholders will be benefited from it, the questions that need to be answered are:

(i) As the sole responsible for any investment decision on the Smart Grid are the DSOs, should they engage a Telco/ICT solution provider to procure smart grid services or do it on their own?

(ii) What is the most suitable among the available ICT approaches each time?

New service platforms must be installed exploiting Service Oriented Architecture (SOA) to support core grid services such as remote monitoring and control. The initiating approach seems to be the deployment of an ICT infrastructure capable of supporting critical services so that the continuity of DSO services under critical situations is guaranteed. The higher the availability required the higher the cost of investment.

\subsubsection{Generic Services}

DSOs have a key role in enabling competition in retail markets by facilitating transparent and non-discriminatory access to network and customer information. As competition among retailers, suppliers, and aggregators grows, automated metering approaches enabling fast and reliable information exchange will be required and new activities and services related to dynamic DR and micro-generation will be involved. At present, since the majority of traditional and new stakeholders are not familiar with the Smart Grid concept, it is difficult to accurately define the associated services, formulate detailed ICT requirements and develop accompanying standards. Anyway, it is not clear to DSOs how Telcos and ICT solution providers will come up with platforms and solutions available to all the energy market stakeholders. The stakeholders in the complex electricity supply chain, namely governments, regulators, network operators, network users - both generators and consumers - network equipment manufacturers, consultants, suppliers of household appliances and ICT solutions, and various service providers should engage in the forthcoming competitive environment to facilitate the successful deployment of the Smart Grid and enjoy its economic, functional and environmentally benign operation. The complexity of the power supply chain is high and it might become significantly higher as new technological and economic aspects are identified. Indicatively, in the UK and Germany, a separate market role is given for the metering service providers - also responsible for the "smart meter role out"-increasing the necessity as well as the complexity for cooperation in the energy market/grid and possibly putting new organizational obstacles [27].

\subsection{DSO Business Models Related to the Smart Grid}

Current regulatory frameworks [26] require that DSOs operate as asset managers deriving income from tariffs usually regulated at national level. These tariffs set the cost of transporting electric power across the power network and delivering it to the end users and are usually flat-rate, i.e., independent of time of day, end use and capacity.

Upon completion of the smart grid roll-out, DSOs will have to apply new business models. One such model considers DSOs as energy transport service providers (ETSP). This activity might involve services encompassing consumers, businesses, distributed energy resources (feeding locally generated 
energy into the grid), and transportation (electric vehicle charging). Hence, challenges related to balancing and peak shaving/load management activities will be created, requiring DSOs to analyze operational data and use it to optimize the network investment planning.

New ICT solutions together with relevant standards and protocols are needed to support the concept of prosumer (pro-ducer + con-sumer) and the penetration of home automation. Home energy controlling hubs must be formed that will collect real-time or near real-time data concerning energy consumption from smart household appliances to enable intelligent automation. To implement the new smart-grid-based DSO model, converged broadband communications are necessary for the deployment of IP-based power networks that will enhance energy efficiency and system reliability at a controllable investment rate.

Currently, a lot of DSOs own subsidiaries or business units active in electricity generation, trading and/or supply. Their possible functional separation into a T\&D operator and an energy supplier may create a risk regarding the relation between the DSOs and the retail users as the former may loose their direct relationship with the latter as they may have to allow access to their billing/usage data and be left only with the management of the power grid assets. Taking into account that the Smart Grid employs technologies that allow the end users to indirectly influence the power system operation, such a perspective would pose challenges to DSOs in their role of market facilitation. A careful assessment of how the Smart Grid is related to/affected by the various unbundling models is a prerequisite to establish proper relations among end users, energy suppliers and DSOs.

Many DSOs are investing in aligning their ICT systems to the expected unbundled market structure and the corresponding business models [26]. The relevant projects do not take into account the potential impact of future smart grid developments. There is a risk of misalignment, which, in turn, may lead to unsuccessful investments and potential delays in the deployment of the Smart Grid.

\section{Relating the Smart Grid Benefits to Power System Operation and Business Needs}

Due, first, to the key role of the Smart Grid in enhancing the reliability, efficiency and security of energy supply and, second, to the significant investments required a systematic cost-benefit approach is necessary to assess the benefits expected from a smart grid project and justify its implementation. To be realistic and effective, the relevant analysis should carefully take into account real data from smart grid pilot projects, either past or currently developing [28-30].

Benefits can be regarded as the beneficial effects from the deployment of a smart grid project as assessed by the benefited sectors or entities involved, i.e., the utilities, the end users and the society. In general, the benefits from Smart Grid may differ from one sector to another or between entities belonging to the same sector. The DOE-EPRI cost and benefit methodology [31] attempts to allocate benefits to utilities, consumers, and the society. The various stakeholders in the electricity market will benefit from the Smart Grid unequally but, hopefully, to the desired degree. Utilities will benefit from Smart Grid through improved operation including reduction and proper handling of outages, drastic reduction in technical and non-technical power losses, better asset utilization, automated and more frequent billing, improved system maintenance and network planning and enhanced grid surveillance. Consumers will be benefit through more reliable services, reduced businesses losses, potential bill savings and the ability to almost dynamically adapt their consumption to their needs and/or market 
conditions. The society will benefit from the Smart Grid as it offers improved reliability and security of power delivery and contributes to the reduction of $\mathrm{CO}_{2}$ emissions by facilitating the transition from fossil fuel power generation to renewable energy generation.

In order to exactly determine and quantify the benefits, it is important to realize that the beneficial effects of smart grid projects depend on many project-specific factors, such as the communications platform implemented, the ICT solutions and operational practices adopted, the applications and services provided. Therefore, the first step for the assessment of the relevant benefits is to examine the targets of the specific smart grid project. To go through such an investigation, a proper classification is required of the assets (technologies/operations/systems) involved in a smart grid deployment and of the expected benefits.

A detailed classification of the smart grid benefits with regard to the benefited sectors [28-30] is given in Figure 1. A similar approach adopted to depict the classification of the smart grid benefits with regard to its optimization targets [31-33] is given in Figure 2. The optimization targets should be expressed in terms of measurable parameters either quantified in terms of money or related to specific performance metrics.

It can readily be deduced that a benefit matrix can be formed that associates the various kinds of benefits with the corresponding benefited sectors. In some cases, benefits derived from functions supporting various key operational objectives, e.g. to reduce outages, $\mathrm{CO}_{2}$ emissions, etc., might be complementary. Indicatively, when all consumers react on price signals with steerable devices, such as heat pumps, EV, etc., but the DSO cannot modify the relevant energy streams, severe problems might occur; hence, DSOs have to invest to reinforce grid assets. The approach proposed hereafter is focused on the Distribution and Automation sectors. A more general analysis should take into account complex situations/scenarios, such as how to allocate profits when different market stakeholders are involved, how competitors and regulated entities (DSOs/TSOs) should be dealt with, etc.

Figure 1. Classification of smart grid benefits with regard to the benefited sectors.

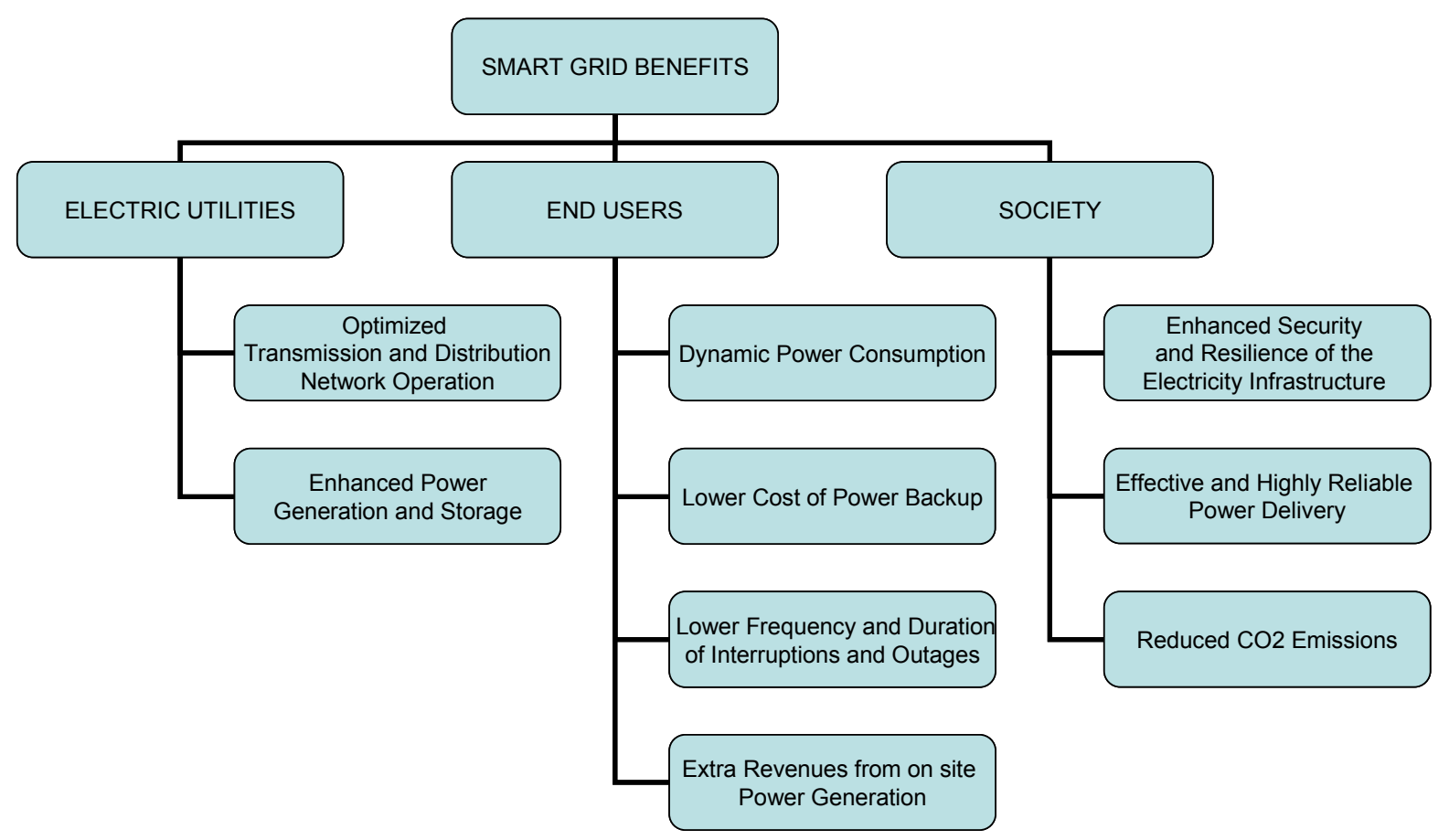


Figure 2. Classification of smart grid benefits with regard to the target sectors involved.

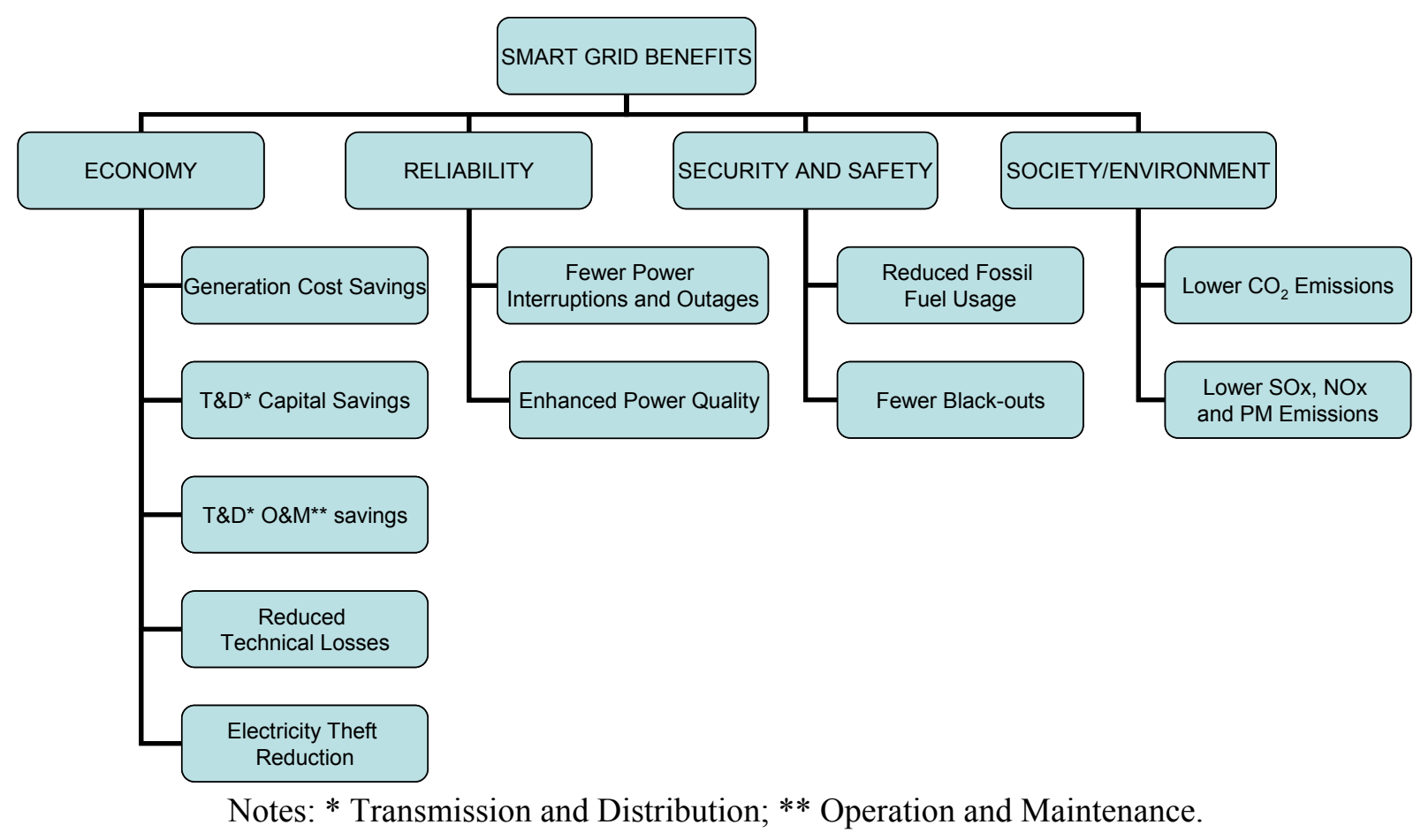

Referring to the benefit matrix, all its entries should be studied in detail in the attempt to model and estimate, preferably in terms of money, the respective benefit of the smart grid and acquire the ability to process the individual benefits under any criterion. Though the application and usefulness of such a matrix may differ from one utility to another, the basic concept of the proposed methodology remains the same. Figure 3 provides a rough schematic mapping of the optimization targets to the benefited sectors of the electricity market stakeholders.

Figure 3. Mapping of optimization targets to benefited sectors.

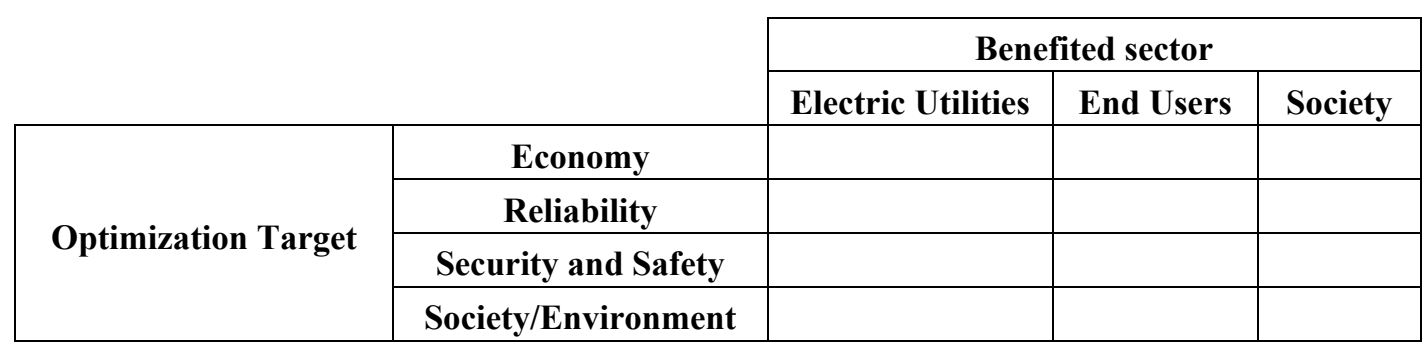

After having identified the key operational requirements and business needs that must be satisfied by a smart grid deployment, the first step of the required techno-economic analysis is to provide a list of the objectives that must be accomplished and relate them to the various smart grid functions. The value of each benefit can be obtained, possibly in terms of maximum annual savings or cost avoidance, based on calculations concerning the improvement achieved with regard to the objectives. The smart grid benefits are associated with the various functions which, in turn, are associated with the various operational requirements and business needs. Therefore, what is important in this procedure is the correlation of two tables, i.e., of one that records the objectives as columns and the functions as rows and of one that records the functions as columns and the benefits as rows. 


\section{Benefits Expected from Improved Distribution Reliability due to Smart Grid Operation}

\subsection{A Study Case}

Based on the methodology presented in Section 4, Section 5 focuses on assessing the aggregate benefit expected from improved distribution reliability due to the Smart Grid. This benefit is referred to by the utilities as one of the primary reasons for implementing Distribution and Automation (DA) interventions [34-38]. Automatic or remotely controlled switches installed on specific nodes of the distribution grid enable utilities to identify and restore faults more quickly. Hence, the time the customers remain without electric power is significantly reduced. As referred to in Sec.4, the specific business need (BN) addressed is to increase distribution reliability [7] and the relevant objectives towards its accomplishment are: (i) $\mathrm{OBJ}_{1}$ : make the outages less in number and shorter in duration and (ii) $\mathrm{OBJ}_{2}$ : reduce the restoration time and cost.

The functions that have been developed to support a wide analysis that aims at estimating the smart grid benefits due to DA interventions (and improvement) are tabulated in Table 1 [28].

The two objectives, $\mathrm{OBJ}_{1}$ and $\mathrm{OBJ}_{2}$, are supported by a different set of the above functions. Each function requires the application of specific technological options, the commercial availability of which affects the respective implementation time and cost. Taking into account real market data, realistic assumptions can be made about the time various technological options will become available within the specified time scale of implementation. When applicable, it is recommended to classify the time schedule into three time horizons: near-term (between 0 and 5 years), mid-term (between 5 and 15 years), and long-term (exceeding 15 years).

The benefits related to increase the distribution reliability are determined based on the changes caused to one or more of the following reliability metrics: System Average Interruption Duration Index (SAIDI); System Average Interruption Frequency Index (SAIFI); and Customer Average Interruption Duration Index (CAIDI). SAIDI is the system average duration of sustained outages and SAIFI is the system average number of sustained outages. CAIDI is the average duration of sustained interruptions per outage event. The proposed methodology takes into account how the transition from the conventional power grid to the Smart Grid affects the above reliability metrics [39-44].

In Table 2 the assumptions concerning the SAIFI improvement of a hypothetical electric power network serving as the study case [45] are given in terms of percentage probability.

In each case the partial SAIFI improvement is evaluated as the intersection of the independent functions involved. The aggregate SAIFI improvement is evaluated as the union probability of the partial SAIFI improvement events. To determine the benefit related to the aggregate SAIFI improvement, one should: (i) translate the latter in minutes of outage or, equivalently, in extra minutes of served energy demand for the various customer groups (home users, corporate users, etc.) and (ii) evaluate the extra revenue offered to the utility. The amount of extra energy sold could constitute a new billable event, i.e. new revenue, for the utility but it can also be viewed as cost avoidance or risk reduction for both the DSOs and the end users involved. Though the evaluation of the former benefit is straightforward, the evaluation of the latter one is complex and depends drastically on the customer group considered each time. The usefulness or gravity of the electric energy for the various customer groups and how 
their business or ordinary life might be affected varies significantly and costs differently as power interruptions or outages affect the economic position of different customer groups unequally.

Table 1. Functions related to DA and supporting to the smart grid.

Functions
Automatic Voltage and VAR Control
(F01)
Automatic Feeder Reconfiguration-
Single-Level (F02)
Automatic Feeder Reconfiguration-
Multi-Level (F03)
Optimum Power Flow Analysis (F04)

Distributed Energy Resources (DER)

Monitoring (F05)

DER Control by Unit (F06)

DER Control by Class (F07)

Automatic Protection Reconfiguration (F08)

Isolation of Higher Impedance Faults (F09)

Automatic Switching-Local (F10)

Automatic Switching - Central (F11)

Automatic Condition-Based

Equipment Maintenance (F12)

Low-Impact Fault Detection (F13)

Automatic Islanding and

Resynchronization (F14)

Real-Time Communications from the Utility to the Customer (F15)

Automatic Phase Load Balancing

Real-Time Communications from the Customer to the Utility (F17).

\section{Benefits}

It can be implemented by a DSO based on operating strategies, or in response to local or regional contingency or outage events. It also includes the ability to adjust or optimize the distribution power factor to reduce losses or achieve specific power factor targets.

Individual feeders can be reconfigured and optimized, including coordinated switching on the primary feeder or its laterals, or with an adjacent feeder. This may be in response to an outage or for peak load control.

Multiple distribution feeders in an area may be reconfigured and optimized, including those with tie points to one or more substations.

Real-time monitoring and analysis enables distribution operators to make decisions concerning system performance, reliability, power quality, losses and asset utilization.

Individual DER units are monitored for status and output; this information is available to the utility staff in near real-time.

Individual DER units are controlled independently by utilities in near real-time to improve distribution system efficiency and performance.

Individual DER units are controlled in groups or classes, either by the utilities or third-party operators, in near real-time.

It addresses circuit loading and two-way power flow issues associated with high DER penetration.

It enables faster isolation of high impedance faults in order to minimize safety hazards and reduce damage to equipment and property.

It is used to isolate faulted segments of distribution circuits to reduce the duration and scope of power outages. It can also reduce the time and effort required for crews to travel between switch positions and operate the devices manually.

Switches will operate automatically in response to signals from a central distribution management system.

Distribution units equipped with sensors that monitor the grid condition and report accordingly increase the reliability and reduce the cost of maintenance.

It reduces the stress on the transmission and distribution infrastructure prolonging their expected lives and reducing equipment failures.

It is fundamental to microgrid operation; it isolates loads within microgrids, and enhances the operating flexibility of the utilities in certain areas.

Utilities can communicate directly with customers in real time to provide information such as price signals, network conditions, restoration times, and safety advice.

It provides real-time measurements of customer consumption and manages the load through Advanced Metering Infrastructure (AMI) supporting customers decisions.

As in F15 
Table 2. SAIFI assumptions and calculations.

\begin{tabular}{|c|c|}
\hline Assumptions Concerning the SAIFI Improvement & $\begin{array}{l}\text { Partial (\%) } \\
\text { SAIFI } \\
\text { Improvement } \\
\end{array}$ \\
\hline \multicolumn{2}{|l|}{ Automatic Switching (F10) } \\
\hline Percentage of SAIFI caused by mainline outages & $\mathrm{P}_{10,1}=50 \%$ \\
\hline Percentage of previously affected customers that do not experience an interruption & $\mathrm{P}_{10,2}=50 \%$ \\
\hline System percentage that employs automatic switching & $P_{10,3}=50 \%$ \\
\hline Percentage of time adjacent feeder is capable of carrying transferable load & $\mathrm{P}_{10,4}=75 \%$ \\
\hline Partial SAIFI improvement due to Automatic Switching $\left(P_{10}=P_{10,1} P_{10,2} P_{10,3} P_{10,4}\right)$ & $P_{10}=9.38 \%$ \\
\hline \multicolumn{2}{|l|}{ Automatic Condition-Based Equipment Maintenance (F12) } \\
\hline Percentage of SAIFI related to distribution equipment failures & $\mathrm{P}_{12,1}=44 \%$ \\
\hline Percentage of equipment failures that are reduced due to early detection & $P_{12,2}=75 \%$ \\
\hline Percentage of equipment that affects SAIFI by monitoring equipment & $\mathrm{P}_{12,3}=75 \%$ \\
\hline $\begin{array}{l}\text { Partial SAIFI improvement due to Automatic Condition-Based Equipment } \\
\left(P_{12}=P_{12,1} P_{12,2} P_{12,3}\right)\end{array}$ & $P_{12}=24.75 \%$ \\
\hline \multicolumn{2}{|l|}{ Low-Impact Fault Detection (F13) } \\
\hline Percentage of SAIFI caused by this type of equipment failure & $\mathrm{P}_{13,1}=0,5 \%$ \\
\hline Percentage of failures reduced by low-impact fault detection & $\mathrm{P}_{13,2}=90 \%$ \\
\hline Percentage of relevant equipment employing this capability & $\mathrm{P}_{13,3}=100 \%$ \\
\hline Partial SAIFI improvement due to Low-Impact Fault Detection $\left(P_{13}=P_{13,1} P_{13,2} P_{13,3}\right)$ & $P_{13}=0.45 \%$ \\
\hline \multicolumn{2}{|l|}{ Automatic Islanding and Resynchronization (F14) } \\
\hline Percentage of customers included in microgrids & $\mathrm{P}_{14,1}=1 \%$ \\
\hline Percentage of outages that would be avoided as a result of microgrid formation & $\mathrm{P}_{14,2}=90 \%$ \\
\hline Percentage of customers located within the microgrid that does not have lines down. & $\mathrm{P}_{14,3}=50 \%$ \\
\hline $\begin{array}{l}\text { Partial SAIFI improvement due to Automatic Islanding and Resynchronization } \\
\left(P_{14}=P_{14,1} P_{14,2} P_{14,3}\right)\end{array}$ & $P_{14}=0.45 \%$ \\
\hline $\begin{array}{l}\text { Aggregate SAIFI improvement } P_{S A I F I}=P_{10}+P_{12}+P_{13}+P_{14}-\left(P_{10} P_{12}+P_{10} P_{13}+P_{10} P_{14}+P_{12} P_{13}+\right. \\
\left.\left.P_{12} P_{14}+P_{13} P_{14}\right)+\left(P_{10} P_{12} P_{13}+P_{10} P_{12} P_{14}+P_{12} P_{13} P_{14}\right)-P_{10} P_{12} P_{13} P_{14}\right)\end{array}$ & $P_{\text {SAIFI }}=32.40 \%$ \\
\hline
\end{tabular}

As an example network owners receive revenue: (i) from connection charges-which are not directly related to energy flow and (ii) from the use-of-system charges - which are related to energy flow. Any attempt to improve system efficiency and reduce the peak load does not necessarily reduce the energy volume. In fact, it is the adaptive energy shifting that makes the aggregate use of energy more efficient. Heat pumps and electrical vehicles are both applications aiming at shifting from fossil fuels to electricity but, at the same time, leading to increased electricity demand, though to a lower overall primary energy consumption. Reducing energy consumption by improving efficiency is a sustainable way of contributing towards achieving fundamental targets related to electric power. In many cases current regulatory frameworks provide the regulated organizations with incentives to increase their cost efficiency by reducing their operating expenses without, however, ensuring the financial health required by such capital-investment projects. It is questionable whether additional cost reductions can still be achieved in the traditional power grid. Indeed, further pressure on cost reductions may result in severe QoS loss. An alternative way to compensate for the above trade-off would be a new regulatory framework that incentivizes the implementation of the Smart Grid and allows DSOs to recover their investments at a market rate. The final price paid by customers should 
differentiate the energy price component and the grid access tariff component, the last one consisting of the transmission and distribution costs as well as other regulated costs [46].

A reasonable assumption could be that the remuneration of distribution and transmission companies, viewed as regulated entities, should not be determined solely from the volatility in energy demand but should reflect the development, proper maintenance and efficient operation of their networks and their components. A careful analysis is recommended concerning the market effects on the network operators in their attempt to make investments on smart grids. To translate the benefit into money, specific macroeconomic measurements and estimations must be employed. The indicative study case presented in Table 2 [7] is summarized in Table 3 wherefrom it is conclude that the change in SAIDI is translated into a significant average increase in time of operation per year per customer.

Table 3. Benefits due to less power outages.

\begin{tabular}{cccccc}
\hline $\begin{array}{c}\text { Current SAIFI } \\
\text { (\# outages) }\end{array}$ & 1.066 & $\begin{array}{c}\text { Change in } \\
\text { SAIFI (\%) }\end{array}$ & 32.4 & $\begin{array}{c}\text { New SAIFI } \\
\text { (\# outages) }\end{array}$ & 0.721 \\
$\begin{array}{c}\text { Current SAIDI } \\
\text { (min.) }\end{array}$ & 108.3 & $\begin{array}{c}\text { Change in } \\
\text { SAIDI (\%) }\end{array}$ & 32.4 & $\begin{array}{c}\text { New SAIDI } \\
\text { (min.) }\end{array}$ & 73.2 \\
\hline
\end{tabular}

Knowing the number of customers, their tariffs and their utility functions, the aggregate benefit due to SAIDI improvement can be evaluated in terms of money.

\subsection{Guidelines for the Assessment of a Smart Grid Investment}

Translating benefits into money is essential for an investment decision and must be based on the aggregate beneficial change brought about by the smart grid project [47-53]. Therefore, to evaluate and compare past, present and future values, a baseline (or reference) scenario is necessary. Referring to the study case presented in Section 5.1 that aims at assessing the operating net cash flows generated by deploying the smart grid infrastructure to increase distribution reliability, one may consider the number of minutes of unserved energy demand in the year prior to the beginning of smart grid operation as the reference scenario and evaluate the subsequent increase in sales and/or savings on this basis. To measure the change caused by the transition to smart grid operation, two scenaria, namely the baseline scenario and the roll-out one, must be studied. The objective of the former is to give the actual state of the power system prior to the deployment of the smart grid project whereas the objective of the latter is to describe the state of the power system during and after the deployment of the smart grid project. The two latter states can be readily determined as they can be accurately measured based on the improved operation implemented by the Smart Grid. On the other hand, assessing the baseline scenario might be difficult in practice since the baseline state can not always be measured accurately. Therefore, the baseline scenario is often based on historical data or interpolations of relevant measurements. It is important that both the baseline and the roll-out scenaria are conducted within a specific time duration.

Having determined the baseline and roll-out scenaria, the economic analysis needs to collect and process the information required to translate the smart grid benefits into money. Depending on the benefit, this information might be in the form of raw data, such as hour of served energy demand, or in 
analytic form, such as line losses at transmission and distribution level. All kinds of data must be expressed in terms of units (e.g., in KWh), which could be easily translated into money.

The cost data to process should, at least, comprise the following: annual generation cost, price of a peaking generator, cost of ancillary services, distribution maintenance cost, distribution operation cost, technical losses, percentage of electricity theft, outage time, value of lost load, meter reading cost, restoration cost, $\mathrm{CO}_{2}$ emissions cost.

Though the above cost elements may vary in the future, the QoS for all stakeholders should be improved at low cost. As most DSOs view the active grid management as a complementary action to network enhancement, they must intensify their engagement in smart grid projects in order to assure improved QoS to customers and because of their prominent role in system security. Certainly, governments and regulatory bodies have to work together towards an optimized retail market model encompassing all participants in the value chain, from generators to consumers, in order to minimize the total cost.

As a result of increased interaction among the electricity market stakeholders, the data flows that DSOs will have to manage are expected to increase. The consumers should be properly informed and trained to retrieve and process the available information to acquire the related benefits. The cost of implementing a smart grid solution or functionality should always be lower than the cost of a conventional solution or the cost of doing nothing. It is important to take into account that system operators may have to invest and develop the smart grid infrastructure in favor of other agents. Therefore, both the aggregate cost/benefit of each smart grid solution and the cost/benefit for each stakeholder should be properly analyzed and estimated.

As previously mentioned, a specific benefit is quantified by the difference of the respective values after (roll-out scenario) and before (baseline scenario) the implementation of a smart grid project. The value of a benefit in terms of money is given by:

$$
\text { Benefit }(\$ \text { or } €)=[\text { Value }]_{\text {roll-out }}-[\text { Value }]_{\text {baseline }}
$$

After translating the benefits into money, it is important to determine the net present value (NPV) of a smart grid project. NPV results from subtracting A from B, where A is the capital expenditure (CAPEX) required to implement the smart grid project brought to the present time and $\mathrm{B}$ is the present value of the incremental operating cash flows generated due to the smart operation of the power grid. To evaluate the present value of future operating cash flows, which express the annual long run incremental costs of operation (OPEX, operational expenditure) and revenues, the relevant amounts of money are discounted by a discount interest and summed. The CAPEX components are related to investments for procuring capital assets supportive of generation, transmission and distribution, operation and maintenance, ancillary services, equipment controlling $\mathrm{CO}_{2}, \mathrm{SO}_{2}, \mathrm{NO}_{\mathrm{x}}$, emissions, operation and permits, etc. In general, the CAPEX components are annual and reflect the budget available/required to acquire the capital assets for setting up an investment, in this case a smart grid project. The investment is financially assessed for a longer period of time. Based on the above considerations, the NPV of a smart grid investment is given by:

$$
\operatorname{NPV}(\$ \text { or } €)=\sum_{i=1}^{N} \frac{O C F_{i}}{(1+r)^{i}}-\sum_{i=0}^{K} \frac{C A P E X_{i}}{(1+r)^{i}}
$$


where $N$ is the number of years within which the particular smart grid project is evaluated; $K$ is the number of years within which the capital investment in the smart grid project is expected to take place $(K<N)$; OCF stands for Operating Cash Flows (OCF = EBIT - Taxes + Depreciation, where EBIT stands for Earnings Before Interests and Taxes. In the case of a smart grid project, earnings represent the money saving due to the project implementation and can be evaluated by summing net new revenues and cost reductions); $r$ is the discount interest (usually equal to the Weighted Average Cost of Capital).

The NPV given by (2) can assist the decision making on whether and how to finance a smart grid investment. Large NPV values favor the initiation of the smart grid project under consideration. A negative NPV implies a careful reconsideration of the smart grid project, unless it leads to an immediate rejection. If the NPV is close to zero, reconsidering and possibly redesigning the project is strongly recommended. A critical factor in determining the NPV is the discount interest. It is usually recommended that this interest be the Weighted Average Cost of Capital (WACC), i.e., a weighted average of equity and debt, reflecting the cost of financing such a project from various funding sources after having incorporated a reasonable return for the shareholders. The WACC can be determined either as pre (including taxes) or post (excluding taxes) value or in real (i.e., without inflation) or nominal (with inflation) terms. As investments will act catalytically toward the development of the Smart Grid, policy makers should formulate a clear mandate and provide clear incentives to stimulate investments in the grid infrastructure. Market definitions and roles and many aspects of the regulatory framework must be reconsidered from a long-term, forward-looking perspective. As the various participants in the value chain of the energy market will enjoy cumulative benefits from the Smart Grid regulatory flexibility is necessary. Finally, a sensitivity analysis that takes into account how the NPV is affected by the various smart grid parameters must be conducted so that the project evaluators are able to assess the alternatives available.

\section{Conclusions}

The present paper proposes a generic framework for the assessment of the benefits expected from the Smart Grid. The basic concept of the Smart Grid and its effect on the electricity market stakeholders are outlined together with the new business models that deserve the consideration of policy makers and regulators. The benefits expected from the Smart Grid are classified according to the various stakeholders with emphasis on DSOs and the end users. A methodological framework for the evaluation of the smart grid benefits is proposed based on the idea of relating the various categories of benefited sectors to the optimization targets related and identifying the particular grid functions that are affected. An indicative study case related to distribution and automation is presented to demonstrate the proposed framework. Finally, the net present value method is applied to assess the investment in a smart grid project based on the benefits it offers, the CAPEX it requires and the cost of its financing. The framework of WACC evaluation and its implications to the assessment of a smart grid project can be the objective of further research. Investing in the Smart Grid is expected to yield significant benefits to the electricity market stakeholders. 


\section{References}

1. Kumar, R.; Ray, P.D.; Reed, C. Smart Grid: An Electricity Market Perspective. In Proceedings of IEEE Power and Energy Society (PES) Conference on Innovative Smart Grid Technologies (ISGT 2011), Anaheim, CA, USA, 17-19 January 2011.

2. Lesourd, J.B. Electricity: The Limits of Commodity Status. Communication. In Proceedings of Conférence sur L'ouverture des Marchés de L'éléctricité, Marseille, France, 23 January 2004.

3. Ruff, L.E. Competitive Electricity Markets: Why They Are Working and How to Improve Them; Report for Harvard Electricity Policy Group, Cambridge, MA, USA, 12 May 1999.

4. Fang, X.; Misra, S.; Xue, G.; Yang, D. Smart Grid-The New and Improved Power Grid: A Survey. IEEE Commun. Surv. Tutor. 2012, 14, 944-980.

5. The Smart Grid: Opportunities for Industry and How IEEE Can Help. Available online: http://www.ieee.org/documents/ieee_smart_grid_whitepaper_sept2011.pdf (accessed on 28 January 2013).

6. Sarafi, A.M.; Tsiropoulos, G.I.; Cottis, P.G. Hybrid Wireless-Broadband over Power Lines: A Promising Broadband Solution in Rural Areas. IEEE Commun. Mag. 2009, 47, 140-147.

7. Power Systems Engineering Research Center. U.S. Energy Infrastructure Investment: Large-Scale Integrated Smart Grid Solutions with High Penetration of Renewable Resources, Dispersed Generation, and Customer Participation. Available online: http://www.pserc.wisc.edu/documents/ publications/papers/2009_general_publications/pserc_smart_grid_white_paper_march_2009_ad obe7.pdf (accessed on 28 January 2013).

8. European Commission; Joint Research Centre (JRC); Institute for Energy and Transport; US Department of Energy-DOE; Office of Electricity and Energy Reliability. Assessing Smart Grid Benefits and Impacts: EU and U.S. Initiatives; Joint Report, 2012. Available online: http://ses.jrc.ec.europa.eu/assessing-smart-grid-benefits-and-impacts-eu-and-us-initiatives (accessed on 28 January 2013).

9. National Energy Technology Laboratory. Understanding the Benefits of the Smart Grid. 18 June 2010. Available online: http:/www.netl.doe.gov/smartgrid/referenceshelf/whitepapers/ 06.18.2010_Understanding\%20Smart\%20Grid\%20Benefits.pdf (accessed on 28 January 2013).

10. Yu, F.R.; Zhang, P.; Xiao, W.; Choudhury, P. Communications systems for grid integration of renewable energy resources. IEEE Netw. 2011, 25, 22-29.

11. Kim, Y.; Thottan, M.; Kolesnikov, V.; Wonsuck, L. A Secure Decentralized Data-Centric Information Infrastructure for Smart Grid, IEEE Commun. Mag. 2010, 48, 58-65.

12. Metke, A.R.; Ekl, R.L. Security technology for smart grid networks. IEEE Trans. Smart Grids 2010, 1, 99-107.

13. Hart, D.G. Using AMI to realize the smart grid. In Proceedings of IEEE Power and Energy Society General Meeting 2008-Conversion and Delivery of Electrical Energy in the 21st Century, Pittsburgh, PA, USA, 20-24 July 2008; pp. 1-2.

14. Roozbehani, M.; Dahleh, M.; Mitter, S. Dynamic pricing and stabilization of supply and demand in modern electric power grids. IEEE Smart Grid Comm. 2010, 10, 543-548.

15. Casazza, J.; Delea, F. Understanding Electric Power Systems: An Overview of the Technology and the Marketplace; John Wiley and Sons, Inc.: Hoboken, NJ, USA, 2003; ISBN 0-471-44652-1. 
16. Rahimi, F.; Ipakchi, A. Demand response as a market resource under the smart grid paradigm. IEEE Trans. Smart Grid 2010, 1, 82-88.

17. Van Werven, M.J.N.; Scheepers, M.J.J. Dispower: The Changing Role of Energy Suppliers and Distribution System Operators in the Deployment of Distributed Generation in Liberalized Electricity Markets. ECN-C-05-048; June 2005. Available online: http://www.ecn.nl/docs/library/ report/2005/c05048.pdf (accessed on 28 January 2013).

18. EURELECTRIC. The Role of DSOs on Smart Grids and Energy Efficiency. A EURELECTRIC position paper; January 2012. Available online: http://www.eurelectric.org/media/26860/ final_the_role_of_dsos_on_sg_ee-13_january_sd-2012-030-0025-01-e.pdf (accessed on 28 January 2013).

19. Nykamp, S.; Andor, M.; Hurink, J.L. Standard incentive regulation hinders the integration of renewable energy generation. Energy Policy 2012, 47, 222-237.

20. Bauknecht, D. Incentive Regulation and Network Innovations; EUI Working Paper RSCAS 2011/02; European University Institute, Florence, Robert Schuman Centre for Advanced Studies, Loyola de Palacio Programme on Energy Policy. Available online: http://cadmus.eui.eu/handle/ 1814/15481 (accessed on 28 January 2013).

21. Gungor, V.C.; Lambert, F.C. A Survey on communication networks for electric system automation. Comput. Netw. 2006, 50, 877-897.

22. Jih, S.; Yin, M.-L. An Availability Analysis on SONET Ring Networks in Power Grid Communications. In Proceedings of Annual Reliability and Maintainability Symposium (RAMS), Reno, NV, USA, 23-26 January 2012.

23. Lazaropoulos, A.G.; Cottis, P.G. Capacity of overhead medium voltage power line communication channels. IEEE Trans. Power Deliv. 2010, 25, 723-733.

24. Lazaropoulos, A.G.; Cottis, P.G. Broadband bia underground medium voltage power lines-Part II: Capacity. IEEE Trans. Power Deliv. 2010, 25, 2425-2434.

25. Gungor, V.C.; Sahin, D.; Kocak, T.; Ergüt, S.; Buccella, C.; Cecati, C.; Hancke, G.P. Smart grid technologies: Communication technologies and standards. IEEE Trans. Ind. Inf. 2011, 7, 529-539.

26. European Commission. Electricity Utilities and Telecom Companies. In Proceedings of 2nd EC-Utilities-Telecom Workshop, Brussels, Belgium, 5 October 2011.

27. European Landscape Report 2012. Available online: http://www.smartregions.net/default.asp? SivuID=26927 (accessed on 29 January 2013).

28. Blazewicz, S.; Shlatz G.; Small, F.; Tobias, S.; Bean, J. (Navigant Consulting Inc). 2008. The Value of Distribution Automation; California Energy Commission, PIER Energy Systems Integration Program, CEC-500-2007-103. Available online: http://www.ilgridplan.org/Shared \%20Documents/CEC\%20PIER\%20Report\%20-\%20The\%20Value\%20of\%20Distribution\%20 Automation.pdf (accessed on 28 January 2013).

29. Sullivan, M.; Schellenberg, J. How to Assess the Economic Consequences of Smart Grid Reliability Investments; Report for Miles Keogh National Association of Regulatory Utility Commissioners, Washington, DC, USA, 29 November 2010.

30. Baer, W.S.; Fulton, B.; Mahnovski, S. Estimating the Benefits of the Grid Wise Initiative; Report for the Pacific Northwest National Laboratory, Richland, WA, USA, 2004; TR-160-PNNL. 
31. Electric Power Research Institute (EPRI). Methodological Approach for Estimating the Benefits and Costs of Smart Grid Demonstration Projects; Final Report; U.S. Department of Energy, Electric Power Research Institute: Palo Alto, CA, USA, January 2010. Available online: http://www.smartgridnews.com/artman/uploads/1/1020342EstimateBCSmartGridDemo2010_1_.ppd (accessed on 28 January 2013).

32. Electric Power Research Institute (EPRI). Estimating the Costs and Benefits of the Smart Grid: A Preliminary Estimate of the Investment Requirements and the Resultant Benefits of a Fully Functioning Smart Grid; 2011 Technical Report; U.S. Department of Energy, Electric Power Research Institute: Palo Alto, CA, USA, March 2011. Available online: http://www.sgiclearinghouse.org/node/3272 (accessed on 28 January 2013).

33. Electric Power Research Institute (EPRI). The Cost of Power Disturbances to Industrial and Digital Economy Companies; Submitted to EPRI's Consortium for Electric Infrastructure for a Digital Society, (CEIDS); 29 June 2001. Available online: http://www.onpower.com/pdf/ EPRICostOfPowerProblems.pdf (accessed on 28 January 2013).

34. Elmakias, D. Reliability of distribution systems. Stud. Comput. Intell. 2008, 111, 373-404.

35. Elmakias, D. Basic Notions of power system reliability. Stud. Comput. Intell. 2008, 111, 1-53.

36. Moslehi, K.; Kumar, R. Smart Grid-A Reliability Perspective Paper. In Proceedings of IEEE Power and Energy Society (PES) Conference on "Innovative Smart Grid Technologies", Washington, DC, USA, 19-20 January 2010.

37. McGranaghan, M.; Goodman, F. Technical and system requirements for advanced distribution automation. In Proceedings of 18th International Conference and Exhibition on Electricity Distribution, Turin, Italy, 6-9 June 2005; pp. 1-5.

38. Tumilty, R.M.; Elders, I.M.; Burt, G.M.; McDonald, J.R. Coordinated protection, control \& automation schemes for microgrids. Int. J. Distrib. Energy Resour. 2007, 3, 225-241.

39. Allan, R.N.; Borkowska, B.; Grigg, C.H. Probabilistic analysis of power flows. Proc. Inst. Electr. Eng. 1974, 121, 1551-1556.

40. Dopazo, J.F.; Klitin, O.A.; Sasson, A.M. Stochastic load flows. IEEE Trans. Power Appl. Syst. 1975, 94, 299-309.

41. Anderson, R.N.; Boulanger, A.; Powell, W.B.; Scott, W. Adaptive stochastic control for the smart grid. Proc. IEEE 2011, 99, 1098-1115.

42. Bu, S.; Yu, F.R.; Liu, P.X. Stochastic unit commitment in smart grid communications. In Proceedings of IEEE 2011 International Conference on Computer Communications INFOCOM) Workshop on Green Communications and Networking, Shanghai, China, 10-15 April 2011; pp. 307-312.

43. He, M.; Murugesan, S.; Zhang, J. Multiple timescale dispatch and scheduling for stochastic reliability in smart grids with wind generation integration. In Proceedings of IEEE International Conference on Computer Communications (INFOCOM) Mini-Conference, Shanghai, China, 10-15 April 2011; pp. 461-465.

44. Parvania, M.; Fotuhi-Firuzabad, M. Demand response scheduling by stochastic SCUC. IEEE Trans. Smart Grid 2010, 1, 89-98. 
45. National Energy Technology Laboratory. Building a Smart Grid Business Case; Report for the U.S. Department of Energy Office of Electricity Delivery and Energy Reliability, Palo Alto, CA, USA, August 2009.

46. EURELECTRIC Comments on ERGEG Position Paper on Smart Grids. A EURELECTRIC position paper. March 2010. Available online: http:/www.eurelectric.org/media/43983/ eurelectric_pp_ergeg_smart_grids_final-2010-230-0001-01-e.pdf (accessed on 28 January 2013).

47. LaCommare, K.H.; Eto, J.H. Cost of Power Interruptions to Electricity Consumers in the United States (U.S.); LBNLL: Berkeley, CA, USA, 2006.

48. De Nooij, M.; Koopmans, C.; Bijvoet, C. The value of supply security: The costs of power interruptions: Economic input for damage reduction and investment in networks. Neth. Energy Econ. 2007, 29, 277-295.

49. Giordano, V.; Onyeji, I.; Fulli, G.; Jimenez, M.S.; Filiou, C. Guidelines for Conducting a CostBenefit Analysis of Smart Grid Projects; Report No. EUR 25246 EN. Available online: http://ec.europa.eu/energy/gas_electricity/smartgrids/doc/20120427_smartgrids_guideline.pdf (accessed on 28 January 2013).

50. Bouhouras, A.S.; Labridis, D.P.; Bakirtzis, A.G. Cost/worth assessment of reliability improvement in distribution networks by means of artificial intelligence. Electr. Power Energy Syst. 2010, 32, 530-538.

51. Chun-Lien, S.; Jen-Ho, T. Outage costs quantification for benefit-cost analysis of distribution automation systems. Int. J. Electr. Power Energy Syst. 2007, 29, 767-774.

52. Bushnell, J.B.; Soft, S.E. Improving private incentives for electric grid investment. Resour. Energy Econ. 1997, 19, 85-108.

53. Kalagnanam, G.J.; Katz, D.; Squillante, M.; Zhang, X.; Feinberg, E. Incentive design for lowest cost aggregate energy demand reduction. In Proceedings of 2010 First IEEE International Conference on Smart Grid Communications (SmartGridComm), Yorktown Heights, NY, USA, 4-6 October 2010; pp. 519-524.

(C) 2013 by the authors; licensee MDPI, Basel, Switzerland. This article is an open access article distributed under the terms and conditions of the Creative Commons Attribution license (http://creativecommons.org/licenses/by/3.0/). 